\title{
Acute Effects of Air Pollution on Human Health in China: Evidence and Prospects
}

The adverse impacts of air pollution on human health have already been a major environmental issue globally, and the challenges are even more severe in China. The World Health Organization (WHO) estimates that 4.2 million deaths annually can be attributed to outdoor air pollution in 2016 (1). For example, ambient fine particulate matter $\left(\mathrm{PM}_{2.5}\right)$ has been ranked as the fourth leading risk factor for disease in China (2). Atmospheric ozone $\left(\mathrm{O}_{3}\right)$ pollution has demonstrated an increasing trend year by year, and the problem of regional compound pollution has become prominent. Therefore, the acute health hazard of air pollution is a major environmental, medical, and public health burden in China. Clarifying the exposure-response relationships between complex air pollution and population-based acute health effects are great strategic demands. With funding from the National Key Research and Development Program of the Ministry of Science and Technology, the "China Short-Term Health Effects of Air Pollution Study" (China SHEAP Study) project was carried out in 2016 and hosted by the National Institute of Environmental Health (NIEH) of China CDC. This project gathered domestic research and development institutions specialized in national public health and clinical medicine to jointly undertake the task. During the four-year implementation period, the project has achieved a series of innovative findings: 1) it clarified the acute impact of short-term exposure of air pollutants on death of residents and their spatiotemporal distribution characteristics in 106 regions nationwide; 2) it systematically evaluated the acute exposure-response relationships between particulate matter (PM) as well as gaseous pollutants and death, morbidities and symptoms of respiratory and cardiovascular diseases in typically polluted cities from three regions and ten urban agglomerations that conducted national air pollution prevention and control; 3) identified acute health effects of differential particle size and chemical component exposures on children, healthy adults, and patients with cardiopulmonary diseases and determined the particles and their components with major toxicity potency; and 4) conducted intervention research to assess the impact of different intervening measures on acute health hazards of air pollution. Through multilevel and multidimension data as well as the integration of key technologies, this project illustrated the distribution characteristics of population-based acute health risks of complex air pollution and established a dataset for complex air pollution and health as well as a data integration platform.

In this special issue, we invited colleagues and collaborators involving in the China SHEAP study project to report their latest findings. For example, Niu et al. examined the associations between air pollutants with daily hospitalizations and outpatient visits of chronic obstructive pulmonary disease (COPD) and asthma within 5 Chinese cities. The results showed that air pollutants were significantly related to the increasing outpatient and hospitalization rates of chronic respiratory diseases (3). Liu et al. assessed the associations of short-term $\mathrm{PM}_{2.5}$ mass exposure with several ambulatory blood pressure (BP) monitoring indicators from a panel study conducted in Beijing, Shanghai, Wuhan, and Xi'an. The results indicated that short-term $\mathrm{PM}_{2.5}$ exposure was significantly associated with BP elevations (4). Xia and Niu et al. evaluated the associations between personal $\mathrm{O}_{3}$ exposure and biomarkers of inflammation, oxidative stress, and mitochondria oxidative damage among 43 college students in Shanghai. They found that short-term exposure to low concentrations of $\mathrm{O}_{3}$ was significantly associated with vascular inflammation, lipid peroxidation, and mitochondrial oxidative damage (5). By conducting a randomized crossover study in Beijing, Zhang et al. found that short-term co-exposure to multiple ambient pollutants could disturb the cardiac autonomic function, and that black carbon (BC) and noise were the two pollutants with the greatest contribution (6). Finally, Chen et al. applied two time-series approaches with a two-stage statistical analysis to estimate whether and how temperature-modified acute effects of $\mathrm{O}_{3}$ affected mortality in Beijing Municipality, Tianjin Municipality, Hebei Province, and surrounding areas. The results suggested that short-term exposure to $\mathrm{O}_{3}$ was significantly associated with the increased risk of mortality and that the association was positively modified by relative higher ( $>75$ th 24 h-average temperature) or extreme cold temperature $(<10$ th 24 h-average temperature) $(7)$.

In summary, the above studies employed different epidemiological designs to assess the impact of short-term air pollution exposure on multidimensionally adverse health outcomes. These provocative findings warrant a 
multiomics approach to comprehensively explore the etiological evidence, the underlying mechanisms, and the causal linkages between air pollution and health effects in China, relying on the available list of biomarkers associated with key toxic components of air pollutants. In view of the compound characteristics of air pollution complex in China, establishing innovative technologies and methods for health risk assessments of multiple exposures simultaneously as well as illustrating their joint effects and mechanisms are highly needed. In addition, the development of early warning techniques for health risks may promote residents to efficiently take actions and protections against air pollution exposures and their health hazards.

doi: $10.46234 / \mathrm{ccdcw} 2021.229$

\# Corresponding author: Xiaoming Shi, shixm@chinacdc.cn.

${ }^{1}$ China CDC Key Laboratory of Environment and Population Health, National Institute of Environmental Health, Chinese Center for Disease Control and Prevention, Beijing, China.

Submitted: October 28, 2021; Accepted: November 02, 2021

\section{REFERENCES}

1. World Health Organization. Ambient (outdoor) air pollution. http://www.who.int/mediacentre/factsheets/fs313/en/. [2018-10-31].

2. GBD 2019 Risk Factors Collaborators. Global burden of 87 risk factors in 204 countries and territories, 1990-2019: a systematic analysis for the Global Burden of Disease Study 2019. Lancet 2020;396(10258):1223 - 49. http://dx.doi.org/10.1016/S0140-6736(20)30752-2.

3. Niu HT, Yu T, Li XX, Wu HN, Yan ML, Duan RR, et al. Exposure response relationship of acute effects of air pollution on respiratory diseases - China, 2013-2018. China CDC Wkly 2021;3(45):943 - 7. http://dx.doi.org/10.46234/ccdcw2021.230.

4. Liu FC, Lin ZN, Wang XY, Yang XL, Liu Q, Xing XL, et al. Impacts of $\mathrm{PM}_{2.5}$ on ambulatory blood pressure monitoring indicators attenuated by blood pressure control status and treatment - Two Cities and Two Municipalities, China, 2017-2019. China CDC Wkly 2021;3(45):948 - 53. http://dx.doi. org/10.46234/ccdcw2021.231.

5. Xia YJ , Niu Y, Cai J, Liu C, Meng X, Chen RJ,et al. Acute effects of personal ozone exposure on biomarkers of inflammation, oxidative stress, and mitochondrial oxidative damage — Shanghai Municipality, China, May-October 2016. China CDC Wkly 2021;3(45):954 - 8. http://dx.doi.org/10.46234/ ccdcw2021.232.

6. Zhang WL, Yang X, Jia X, Dong W, Li HY, Pan L, et al. Co-exposure to multiple pollutants and its cardiovascular effects in a subway system - Beijing Municipality, China, 2017. China CDC Wkly 2021;3(45):959 - 63. http://dx.doi.org/10.46234/ccdcw2021.233.

7. Chen C, Liu J, Shi WY, Li TT, Shi XM. Temperature-modified acute effects of ozone on human mortality — Beijing Municipality, Tianjin Municipality, Hebei Province, and surrounding areas, China, 2013-2018. China CDC Wkly 2021;3(45):964 - 8. http://dx.doi.org/10.46234/ccdcw2021.234.

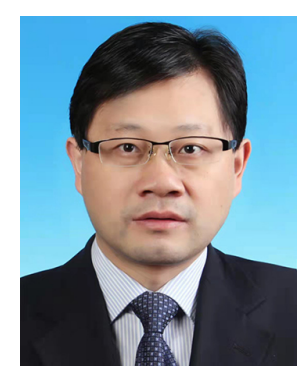

Xiaoming Shi, MD, PhD

Professor and Director, National Institute of Environmental Health (NIEH), China CDC Principal Investigator, National Key Research and Development Program of China, "China Short-term Health Effects of Air Pollution Study (No.2016YFC0206500)" 